\section{Correspondence to 'Hypersensitivity reactions with allopurinol and febuxostat: a study using the Medicare claims data'}

We read with great interest the article by Singh and Cleveland, which the authors reported that the observed hypersensitivity reactions (HSRs) associated with allopurinol and febuxostat were not different. ${ }^{1}$ This conclusion differs from previous studies on HSRs associated with allopurinol and febuxostat using claimed data $^{2}$ and intramural databases. ${ }^{3}$ The discrepancy may arise from different inclusion criteria for the diagnosis of HSRs based on International Classification of Diseases, Ninth Revision (ICD-9) coding system, methods for identifying causative drugs, methods for stratification, as well as ethnicity of the involved population.

The definition of HSR in the study by Singh and Cleveland adopted ICD-9 diagnostic codes specifying eosinophilia (288.3), HSR- associated arthropathy (713.6), anaphylactic reactions (995.0) or unspecified adverse drug effect (995.2) or allergy (995.3), plus the baseline exclusion for E930 to E949. ${ }^{1}$ On the contrary, in our previous studies, the definition of cutaneous adverse reactions (CARs) using the Taiwanese registry database involved drug-induced dermatitis (693.0), erythema multiforme (695.1) or erythematous conditions (695.89 or 695.9)..$^{2-4}$ Likewise, another study based on intramural database and metaanalysis defining severe CARs as 693, 695.1 or 695.9/695.89 also suggested that the overall incidence of febuxostat-associated HSR was lower than that of allopurinol (0.2 vs 2.7 per 1000 users; $\mathrm{p}<0.001)$, with regard to these dermatological manifestations. ${ }^{3}$ From a clinical standpoint, as allopurinol-associated HSRs or CARs are delayed type of hypersensitivity, which normally involve the onset of maculopapular eruption, drug reactions with eosinophilia and systemic symptoms, or StevensJohnson syndrome/toxic epidermal necrolysis, ${ }^{56}$ defining HSR with ICD-9 codes $713.6,995.0$ or 995.2 , should be not appropriate. This suggests that the discrepancy between these studies could be attributed to the inclusion criteria for the diagnosis of HSRs.

Previous studies have pointed out that colchicine rarely causes HSR, as its notoriety score in the algorithm of drug causality for epidermal necrolysis (ALDEN) is $\mathrm{zero}^{7}$; the main reason of colchicine being reported as HSR-associated drugs would be due to the fact that it is oftenly used in conjunction with allopurinol. ${ }^{8}$ Likewise, without further algorithms to confirm drug causality among these ICD-9 coding-based analyses, ${ }^{1}{ }^{2}$ patients with allopurinol-associated HSRs who have been shifted to febuxostat treatment would also lead to the conclusion of febuxostat being counted as the culprit drug. Apart from HSR-inducing gout or hyperuricaemia medications such as allopurinol and febuxostat, ${ }^{1-3}$ frequently prescribed drugs like non-steroidal anti-inflammatory drugs (NSAIDs) are commonly involved in HSR as well. ${ }^{9}$ As NSAIDs are widely used and are available over the counter, most of these epidemiological studies on HSR could not rule out concurrent use of these causative drugs. Without reflective parametrics for instance, chronology, $\mathrm{ALDEN}^{7}$ or Narenjo score with weight of notoriety, ${ }^{10}$ studies based on ICD-9 coding system may not identify the specific causative drug among multiple used drugs. Thus, it may be hard to distinguish the most likely causative medications when considering all the taken drugs as allergens.

Although allopurinol-associated HSR has been suggested to be dose dependent, ${ }^{11} 12$ the underlying mechanisms of febuxostat and colchicine-associated HSRs remain unclear. While Singh and
Cleveland stratified the patients with drug dose cut-offs, ${ }^{1}$ the severity of incident HSRs were not analysed. On the contrary, the other studies either stratified for respective HSR severity but adopted different codes for HSR definition, ${ }^{2}$ or used intramural databases without stratifying the doses of the associated drugs. ${ }^{3}$ Specifically, the conclusion that allopurinol was associated with higher risks of CARs than febuxostat in one of these studies was based on the stratification of severity. ${ }^{2}$ This suggests that the stratification methods may attribute to the observed discrepancies.

Furthermore, the incidence rate of HSR varies among countries. For instance, the incidence rates of HSRs in the USA were estimated to be 23.7 for allopurinol, 30.7 for febuxostat and 25.6 for colchicine, per 1000 person years ${ }^{1}$; whereas in Taiwan, the annual incidence rates were 4.68 per 1000 new allopurinol users for HSR, 2.02 per 1000 new allopurinol users for HSRcaused hospitalisation and 0.39 per 1000 new allopurinol users for HSR-caused mortality, as stratified by severity. ${ }^{4}$

Particularly, the prevalence of HLA-B*58:01 allele, an allopurinol HSR-associated gene, fluctuates among countries ${ }^{13}$; moreover, the association of HLA-B*58:01 with allopurinol-induced HSR varies among populations. ${ }^{14}$ It is possible that the susceptibility to febuxostat and colchicine -associated HSRs differs among ethnicities as well.

For the above reasons, we recommend that the cooperation of bedside diagnostics and laboratory is necessary to discriminate which of the causative drugs induce each hypersensitivity event, as confirmed with algorithms involving chronology, ALDEN or Narenjo score with weight of notoriety score, to compare their incident HSRs.

\section{Kevin Sheng-Kai Ma $\odot$, 1,2,3 James Cheng-Chung Wei $\odot$,,4 Wen-Hung Chung ${ }^{5,6,7}$}

${ }^{1}$ Department of Health Policy and Management, Johns Hopkins University Bloomberg School of Public Health, Baltimore, Maryland, USA

${ }^{2}$ Department of Life Science, National Taiwan University, Taipei, Taiwan

${ }^{3}$ Division of Allergy, Immunology and Rheumatology, Chung Shan Medical University Hospital, Taichung, Taiwan

${ }^{4}$ School of Clinical Medicine, Tsinghua University, Beijing, China

${ }^{5}$ Drug Hypersensitivity Clinical and Research Center, Chang Gung Memorial Hospital, Taipei, Taiwan

${ }^{6}$ Department of Dermatology, Chang Gung Memorial Hospital, Taipei, Taiwan

${ }^{7}$ College of Medicine, Chang Gung University, Taoyuan, Taiwan

Correspondence to Dr. Wen-Hung Chung; chung1@cgmh.org.tw

Contributors All other authors (KSM, JCW and WHC) provided their input by contributing to the conceptualization. KSM and WHC contributed to the editing of the manuscript.

Funding The authors have not declared a specific grant for this research from any funding agency in the public, commercial or not-for-profit sectors.

Competing interests The authors declare no conflict of interest.

Patient and public involvement Patients and/or the public were not involved in the design, or conduct, or reporting, or dissemination plans of this research.

Patient consent for publication Not required.

Provenance and peer review Not commissioned; internally peer reviewed.

(c) Author(s) (or their employer(s)) 2020. No commercial re-use. See rights and permissions. Published by BMJ.

KS-KM and JC-CW contributed equally.

$$
\text { Check for updates }
$$

To cite Ma KS-K, Wei JC-C, Chung W-H. Ann Rheum Dis Epub ahead of print: [please include Day Month Year]. doi:10.1136/annrheumdis-2020-218090

Received 23 May 2020

Accepted 25 May 2020 


\section{(5) Linked}

- http://dx.doi.org/10.1136/annrheumdis-2020-218149

Ann Rheum Dis 2020:0:1-2. doi:10.1136/annrheumdis-2020-218090

ORCID iDs

Kevin Sheng-Kai Ma http://orcid.org/0000-0003-1056-1533

James Cheng-Chung Wei http://orcid.org/0000-0003-0310-2769

\section{REFERENCES}

1 Singh JA, Cleveland JD. Hypersensitivity reactions with allopurinol and febuxostat: a study using the Medicare claims data. Ann Rheum Dis 2020;79:529-35.

2 Lin C-W, Huang W-I, Chao P-H, et al. Risk of cutaneous adverse reactions associated with allopurinol or febuxostat in real-world patients: a nationwide study. Int I Clin Pract 2019:73:e13316.

3 Chen C-H, Chen C-B, Chang CJ, et al. Hypersensitivity and cardiovascular risks related to allopurinol and febuxostat therapy in Asians: a population-based cohort study and meta-analysis. Clin Pharmacol Ther 2019;106:391-401.

4 Yang C-Y, Chen C-H, Deng S-T, et al. Allopurinol use and risk of fatal hypersensitivity reactions: a nationwide population-based study in Taiwan. JAMA Intern Med 2015;175:1550-7.

5 Stamp LK, Day RO, Yun J. Allopurinol hypersensitivity: investigating the cause and minimizing the risk. Nat Rev Rheumatol 2016;12:235-42.
6 Chung W-H, Wang C-W, Dao R-L. Severe cutaneous adverse drug reactions. J Dermatol 2016:43:758-66.

7 Sassolas B, Haddad C, Mockenhaupt M, et al. ALDEN, an algorithm for assessment of drug causality in Stevens-Johnson syndrome and toxic epidermal necrolysis: comparison with case-control analysis. Clin Pharmacol Ther 2010;88:60-8.

8 Nguyen K-D, Tran T-N, Nguyen M-LT, et al. Drug-Induced Stevens-Johnson syndrome and toxic epidermal necrolysis in Vietnamese spontaneous adverse drug reaction database: a subgroup approach to disproportionality analysis. J Clin Pharm Ther 2019:44:69-77.

9 Kowalski ML, Makowska JS, Blanca M, et al. Hypersensitivity to nonsteroidal antiinflammatory drugs (NSAIDs) - classification, diagnosis and management: review of the EAACI/ENDA(\#) and GA2LEN/HANNA*. Allergy 2011;66:818-29.

10 Naranjo CA, Busto U, Sellers EM, et al. A method for estimating the probability of adverse drug reactions. Clin Pharmacol Ther 1981;30:239-45.

11 Yun J, Mattsson J, Schnyder K, et al. Allopurinol hypersensitivity is primarily mediated by dose-dependent oxypurinol-specific T cell response. Clin Exp Allergy 2013:43:1246-55

12 Stamp LK, Taylor WJ, Jones PB, et al. Starting dose is a risk factor for allopurinol hypersensitivity syndrome: a proposed safe starting dose of allopurinol. Arthritis Rheum 2012;64:2529-36.

13 Hung S-I, Chung W-H, Liou L-B, et al. HLA-B*5801 allele as a genetic marker for severe cutaneous adverse reactions caused by allopurinol. Proc Natl Acad Sci U SA 2005;102:4134-9.

14 Hershfield MS, Callaghan JT, Tassaneeyakul W, et al. Clinical pharmacogenetics implementation Consortium guidelines for human leukocyte antigen-B genotype and allopurinol dosing. Clin Pharmacol Ther 2013;93:153-8. 